www.jmscr.igmpublication.org Impact Factor 5.244

Index Copernicus Value: 83.27 ISSN (e)-2347-176x ISSN (p) 2455-0450 crossref DOI: https://dx.doi.org/10.18535/jmscr/v5i1.21

Journal Of Medical Science And Clinical Research

\title{
Imaging In Shoulder Joint Diseases
}

Authors

\section{Dr Akhand Pratap Singh ${ }^{1}$, Dr Dinesh Kumar ${ }^{2}$, Dr Arvind Srivastav ${ }^{3}$, Dr Ashish Verma ${ }^{4}$, Dr Ramvilas $\mathrm{Nag}^{5}$, Dr Suman Chaurasia ${ }^{6}$}

${ }^{1}$ Ex. Resident Dept. of Radiology, Institute of Medical Sciences, BHU, Varanasi

Email: dr.akhand07@gmail.com,8765232233

${ }^{2}$ Ex. Resident Dept. of Orthopaedics, Institute of Medical Sciences, BHU, Varanasi

${ }^{3}$ Prof. Department of Radiodiagnosis, Institute of Medical Sciences, BHU, Varanasi

${ }^{4}$ Asso. Prof. Department of Radiodiagnosis, Institute of Medical Sciences, BHU, Varanasi

${ }^{5}$ Senior Resident Dept. of Orthopaedics, Institute of Medical Sciences, BHU, Varanasi

Email: ramvilasnag@yahoo.in, 9660046869

${ }^{6}$ Ex. Resident Dept. of O\&G, ESIC \& PGIMSR, KK Nagar Chennai

Email: sumanchourasia1987@gmail.com,9941578114

Corresponding Authors

Dr Dinesh Kumar

Ex. Resident, Dept. of Orthopaedics, Institute of Medical Sciences, BHU, Varanasi

Email: oyedinesh@gmail.com,9919101897

\section{Abstract}

Background: MRI of the shoulder continues to develop as an important tool in the assessment of joint and soft tissue pathology. The aim of this study is a prospective descriptive compilation of the role of available imaging modalities in evaluation of different clinical syndromes referable to shoulder joint.

Material \& Methods: The Prospective observational study was carried out on 50 patients in the Department of Radiodiagnosis and Imaging, Institute of Medical Sciences, Banaras Hindu University, Varanasi during period of July 2013 to July 2015. Ethical approval was obtained from the local ethics committee. Informed consent was obtained from each patient, prior to their enrolment in this study. Full history was taken especially about any previous operative procedure, any drug allergy or any absolute contraindication for MRI.

Results: The mean age was 24 years and maximum number of patients affected belong to the age group of 2130 years. There was increased incidence of SLAP lesion in patients having Buford complex (50\%) as compared to patients who were not having Buford complex. An initial suspicion of osteochondritis dissecans existed on MRI but was confirmed only with the visualization of bony fragment on MR arthrography. CT scan, which was performed for confirmation, showed the loose body as well.

Conclusion: MRI and MR Arthrography because of its excellent soft tissue contrast, imaging capability in any desired plane is an ideal modality of choice. In the present study, it could be deciphered that MRI was superior to ultrasound for diagnosing rotator cuff tendon tear. The MR Imaging has effectively replaced all other tests in evaluation of shoulder problems.

Key Words: Shoulder joint, MRI, Rotator cuff pathology, MR Arthrography.

\section{Introduction}

The shoulder joint is the most complex of all synovial articulations in human body, with the greatest range of movements (in six planes). Such degree of unrestricted mobility leads to an attendant instability. A series of complex 
ligaments, tendons and muscles are hence required for achieving the fine balance between mobility and instability Morphological evaluation of these soft tissue and bony structures forms the basis for imaging evaluation of shoulder diseases. ${ }^{1}$

An elaborate array of imaging modalities (x-ray, ultrasonography and MRI) are available for optimum non invasive evaluation of shoulder diseases. In addition, certain newer minimally invasive techniques add to the imaging information and act as problem solving techniques in specific clinical situations. ${ }^{1}$

Several methods are used, including plain radiography, magnetic resonance imaging (MRI), magnetic resonance arthrography (MRA) and ultrasound (US), for the shoulder impingement syndrome and rotator cuff tears. Radiography remains the initial investigation in evaluation of fractures of the shoulder girdle .Radiography is useful in diagnosis and confirming the presence of arthritis, it can detect osteoarthritis associated bony features including osteophytes, subchondral cyst and sclerosis. The technique is also used for monitoring the progression of disease and efficacy of treatment in arthritis. Plain radiographs are also used to diagnose various types of shoulder dislocation. However radiography has major deficiency in imaging of synovial tissue, articular cartilage, ligament, periarticular tendons. ${ }^{2}$

The examination of shoulder pathologies is one of the most common applications of musculoskeletal USG due to high incidence of rotator cuff disorders related to aging and sports activity. With appropriate equipment and skilled hands, this technique provides assessment of rotator cuff pathology with high sensitivity and specificity in diagnosis of both partial and full thickness tears with some specific advantage over MRI imaging, such as higher resolution and the ability to examine tissues in both static and dynamics states. MRI and US have a comparable accuracy for detection of full-thickness rotator cuff tears. MRA and US might be more accurate for the detection of partial-thickness tears than MRI. Given the difference in cost of MR and US, high resolution ultrasound may be the most cost-effective diagnostic method for identification of fullthickness tears in a specialist hospital setting. ${ }^{2}$

With the availability of dedicated surface coils for musculoskeletal imaging, MRI of the shoulder continues to develop as an important tool in the assessment of joint and soft tissue pathology. MRI gives clear views of rotator cuff tears, injuries to the biceps tendon and damage to the glenoid labrum, the soft fibrous tissue rim that helps stabilize the joint. MRI has been found to be an informative non-invasive means of evaluating the shoulder impingement syndrome. The impinging structure and entrapped tissues often can be imaged precisely, allowing specific details of the site and extent of bone and soft tissue disease. MRI permits differentiation of bursitis, tendinitis and complete supraspinatus tear. Joint abnormalities due to trauma and sports related injuries such as tears of ligaments and tendons, infections (such as osteomyelitis), tumors (primary tumors and metastases) involving bones and shoulder joints can also be evaluated precisely by MRI. MR Arthrography is held as an accurate imaging procedure for defining rotator cuff pathology, partial thickness tears and labral /capsule abnormalities in glenohumeral instabilities compared to conventional MR imaging. ${ }^{2}$

The present study is a prospective descriptive compilation of the role of available imaging modalities in evaluation of different clinical syndromes referable to shoulder joint.

\section{Material \& Methods}

The Prospective observational study was carried out on 50 patients in the Department of Radiodiagnosis and Imaging, Institute of Medical Sciences, Banaras Hindu University, Varanasi during period of July 2013 to July 2015. Ethical approval was obtained from the local ethics committee. Informed consent was obtained from each patient, prior to their enrolment in this study. Full history was taken especially about any previous operative procedure, any drug allergy or any absolute contraindication for MRI. 


\section{Inclusion Criteria}

* Patients presenting with painful or unstable shoulder joint with or without other associated symptoms

- Patients with restriction of movement at shoulder joint following trivial trauma

* Patients with trauma to the shoulder joint

\section{Exclusion Criteria}

* Patients who were operated previously on same shoulder

* Patients of suspected shoulder joint infections/ neoplastic pathology

* Any absolute contraindication for MRI such as ferromagnetic implants, pacemaker, aneurysm clips

* Patients who were claustrophobic

Patients presenting with shoulder pain, referred for imaging, were evaluated by Digital radiography (FCR capsula XL II), USG (The ultrasound machine used was Diagnostic Ultrasound, iU22), MRI (1.5 Tesla Superconducting magnet) and, if necessary, MR Arthrography.

\section{Results}

The present study is a prospective observational study including 50 patients, having symptoms referred to the shoulder joint and imaged for eliciting different pathologies related to derangement of shoulder joint. The mean age was 24 years and maximum number of patients affected belong to the age group of 21-30 years (table 1). The most common pathology was shoulder dislocation, Degenerative joint disease was seen mostly in old age patients with some form of trauma to shoulder joint in the past (table 2).

In the present study partial rotator cuff tear was found in $32 \%$ cases while complete tear in $6 \%$ cases (table 3). In most of the cases it was seen on postero-superior aspect of humeral head in initial two axial images from top to bottom up to inferior surface of coracoid process (Table 4). All patients subsequently underwent MR Arthrography; classical Bankart lesion was noted in $45 \%$ (10 cases) while ALPSA, Bony Bankart and GLAD were discerned in $27 \%, 22 \%$ and $6 \%$ respectively (Table 5) .
There was increased incidence of SLAP lesion in patients having Buford complex (50\%) as compared to patients who were not having Buford complex (table 6).

An initial suspicion of osteochondritis dissecans existed on MRI but was confirmed only with the visualization of bony fragment on MR arthrography. CT scan, which was performed for confirmation, showed the loose body as well (table 7).

Table 1: Age distribution in present study

\begin{tabular}{|l|c|c|}
\hline Age (in years) & Frequency & Percent \\
\hline $11-20$ & 12 & 24.0 \\
\hline $21-30$ & 21 & 42.0 \\
\hline $31-40$ & 6 & 12.0 \\
\hline $41-50$ & 5 & 10.0 \\
\hline $51-60$ & 5 & 10.0 \\
\hline $61-70$ & 1 & 2.0 \\
\hline Total & 50 & 100.0 \\
\hline
\end{tabular}

Table 2: Incidence according to pathology elicited in present study $(\mathrm{n}=50)$

\begin{tabular}{|l|c|c|}
\hline Pathology & No. of cases & Percentage \\
\hline Rotator cuff tear & 19 & 38 \\
\hline Shoulder dislocation & 22 & 44 \\
\hline Degenerative disease & 13 & 26 \\
\hline Shoulder impingement & 4 & 8 \\
\hline Adhesive capsulitis & 4 & 8 \\
\hline Osteochondritis dissecans & 2 & 4 \\
\hline
\end{tabular}

Table 3: Rotator cuff tear as depicted on various imaging modalities.

\begin{tabular}{|l|c|c|c|c|c|c|}
\hline \multirow{2}{*}{$\begin{array}{l}\text { Rotator cuff } \\
\text { tear }\end{array}$} & \multicolumn{2}{|c|}{ USG $(\mathrm{n}=50)$} & \multicolumn{2}{c|}{ MRI $(\mathrm{n}=50)$} & \multicolumn{2}{c|}{$\begin{array}{c}\text { MR Arthrography } \\
(\mathrm{n}=26)\end{array}$} \\
\cline { 2 - 7 } & No. & $\%$ & No. & $\%$ & No. & $\%$ \\
\hline Present & 14 & 28 & 19 & 38 & 5 & 19.2 \\
Partial & 11 & 22 & 16 & 32 & 5 & 19.2 \\
Complete & 3 & 6 & 3 & 6 & - & - \\
\hline
\end{tabular}

Table 4: Incidence of Hill Sachs lesion observed on various imaging modalities in patients with shoulder instability

\begin{tabular}{|l|c|c|c|c|}
\hline $\begin{array}{l}\text { Hill Sachs } \\
\text { Lesion }\end{array}$ & $\begin{array}{c}\text { X ray } \\
(\mathrm{n}=22)\end{array}$ & $\begin{array}{c}\text { CT } \\
(\mathrm{n}=6)\end{array}$ & $\begin{array}{c}\text { MRI } \\
(\mathrm{n}=22)\end{array}$ & $\begin{array}{c}\text { MR Arthrography } \\
(\mathrm{n}=22)\end{array}$ \\
\hline Present & 6 & 5 & 17 & 14 \\
\hline Absent & 16 & 1 & 5 & 8 \\
\hline
\end{tabular}

Table 5: Distribution of Bankart lesion and its variants on MRI and MR Arthrography

\begin{tabular}{|l|c|c|}
\hline & MRI (n=22) & $\begin{array}{c}\text { MR Arthrography } \\
(\mathrm{n}=22)\end{array}$ \\
\hline Bankart lesion & 16 & 10 \\
\hline Bankart variants & & 6 \\
\hline ALPSA & 2 & 5 \\
\hline Bony Bankart & 4 & 1 \\
\hline GLAD & 0 & \\
\hline
\end{tabular}


Table 6: Buford complex associated with SLAP lesion, as depicted on imaging

\begin{tabular}{|l|c|c|c|}
\hline & $\begin{array}{c}\text { No. of cases with } \\
\text { Buford complex }\end{array}$ & SLAP & Percentage \\
\hline Present & 4 & 2 & $50 \%$ \\
\hline Absent & 22 & 2 & $10 \%$ \\
\hline
\end{tabular}

Table 7: Osteochondritis dissecans in shoulder joint seen on imaging

\begin{tabular}{|l|l|l|l|}
\hline $\begin{array}{l}\text { Osteochondritis } \\
\text { dissecans }\end{array}$ & CT (n=6) & MRI (n=50) & $\begin{array}{l}\text { MR } \\
\text { Arthrography } \\
(\mathbf{n}=\mathbf{2 6})\end{array}$ \\
\hline Present & 2 & 0 & 2 \\
\hline Absent & 4 & 50 & 24 \\
\hline
\end{tabular}

\section{Discussion}

Shoulder joint pathologies are one of the commonest conditions that are encountered in orthopedic outpatient department. Accurate diagnosis of the condition is must before any decision regarding its management is undertaken. Diagnosis is usually suspected clinically and is confirmed by imaging. The various imaging modalities used are Radiography, Ultrasonography, Computed Tomography scan, Magnetic Resonance Imaging and MR Arthrography. Though MR imaging and MR Arthrography are said to be the gold standard for imaging of the shoulder joint, the use of Ultrasonography and plain radiography has its own role in shoulder joint pathologies.

Subjects of present study belonged to the age range of 17 to 65 years with mean age of 24 years. Maximum patients were young adults falling in the age range of 21-30 years. Zacchilli et al $(2010)^{3}$, in their study, also showed that young age and male gender are risk factors for shoulder joint pathologies especially dislocation. This may be due to the relatively heavy manual work load and a more pro-active utilization of this joint by the male population .In present study as well most patients were either athletes, had sustained trauma or were engaged in some kind of heavy mechanical work.

\section{Shoulder Joint Pathologies \\ Rotator Cuff Tear}

Rotator cuff disorder is one of the most common disorders of the shoulder. It is a common cause of chronic shoulder pain in adults. In present study full-thickness tear appeared on ultrasound as hypoechoic/anechoic defects in the tendon, while partial thickness tears showed hypoechoic discontinuity in tendon fibers. On MRI hyperintense area within the tendon was seen with completely torn fibers in full thickness tears and variably intact tendon fibers in partial thickness tear.

In present study, USG detected 14 out of 50 cases of rotator cuff tears while MRI detected 19 cases. According to Bashir et al (2014) $)^{4}$ MRI is better in assessing rotator cuff tear as compared to USG because MRI shows rotator cuff tendons in axial, coronal and sagittal planes which is not possible with USG; also USG is operator dependent.

MRI was observed to be $80 \%$ sensitive and $100 \%$ specific as compared to MR Arthrography for diagnosing rotator cuff tears. In a study by Lenza et al $(2013)^{5}$, the sensitivity and specificity were $79 \%$ and $98 \%$ respectively for MRI for diagnosing rotator cuff tears.In comparison to MRA, USG was $40 \%$ sensitive and $100 \%$ specific for detecting rotator cuff tear. De Jesus et al $(2008)^{6}$ observed MR Arthrography to be more sensitive and specific than either MRI or ultrasound in diagnosing a rotator cuff tear. In the present study also it was found that for the detection of partial thickness rotator cuff tears MR arthrography was superior to USG and MRI as in MRA, contrast medium insinuates between the torn fibers and insertion site. Two cases of partial tendon tears which were missed by USG and MRI were picked up by MR arthrography.

\section{Shoulder Instability Lesions Hill Sach's Lesion}

In the present study Hill-Sachs lesion was the most common injury (78\%) associated with anterior glenohumeral instability. It consists of bony injury of the posterosuperior humeral head manifesting as cortical bony loss, impaction fracture or associated bone marrow edema in acute cases, which cannot be well appreciated with plain radiography. Kim et al (2013) ${ }^{7}$ found Hill-Sachs lesion to be the commonest lesion $(90.7 \%)$ in patients following anterior shoulder 
dislocation. The our study depicted that Hill Sachs Lesion was better diagnosed on MRI and CT as compared to plain radiography. Cluett et al $(2013)^{8}$ also concluded that Hill Sachs lesion can be seen on MRI and CT-scan, but is difficult to appreciate them on plain radiography. The possible explanation for this is that sagittal, axial and coronal views will demonstrate the lesion better on MRI and CT as compared to plain radiography. Also certain subtle lesions which are overseen due to projections in radiography can be well seen in cross sections

\section{Bankart lesion}

In the present study all cases with Bankart lesion were associated with Hill Sachs lesion. A study by Waldt et al (2005) $)^{9}$ revealed that Bankart lesion is the commonest labral lesion associated with anterior shoulder dislocation. Similarly as in the present study, Kim et al (2013) ${ }^{7}$ found that Hill Sachs lesion was very common in patients with Bankart lesion and the severity of Hill Sachs lesions correlates with the extent of Bankart lesions. Similar results were found in a study by Palmer et al (2002) ${ }^{10}$ in which they have shown the sensitivity of MRI in detecting Bankart lesion of only $46 \%$.

\section{Buford Complex with Shoulder instability}

Cases with Buford complex also had associated shoulder instability showing that Buford complex has relation with shoulder instability. Exact etiology is not clear; however, possible explanation may be in Buford complex there is absent labrum in antero-superior part which deepens the articular surface that predisposes the shoulder for instability.

\section{SLAP Lesion in association with Buford complex}

In present study, 2 out of 4 cases with Buford complex have associated SLAP lesion. In a study by Bents et al $2005^{11}$, they found $83 \%$ of cases with Buford complex with associated SLAP lesion. In their study on 235 cases, 6 had a Buford complex (2.5\%); out of these 6 patients, 5 had a corresponding SLAP lesion (83.3\%). They hypothesized that a Buford complex, with absent anterior labral tissue, may allow abnormal stresses to the superior labrum and biceps and predispose the patient to a possible SLAP lesion.

A conglomerate of imaging modalities were utilized in the present study to various clinical syndromes associated with shoulder joint pathologies. The point that emerged from results in this study with a correlation to contemporary literature, indicates towards the utility of MRI with MR arthrography for the purpose. This modality presumed earlier to be a problem solving tool, appears to have now taken a certain stage with the other modalities being primarily supportive in nature.USG however has a added role in dynamic assessment of the joint especially in cases of per-articular pain.

\section{Conclusion}

Pain around the shoulder joint with or without reduced range of motion is commonly referred to the radiologist for evaluation. MRI and MR Arthrography because of its excellent soft tissue contrast, imaging capability in any desired plane is an ideal modality of choice. In the present study, it could be deciphered that MRI was superior to ultrasound for diagnosing rotator cuff tendon tear. The MR Imaging has effectively replaced all other tests in evaluation of shoulder problems.

\section{References}

1. Stecco, A. Castagna and A Carriero . J Orthop Traumatol. Virtual MR arthroscopy of the shoulder: image gallery with arthroscopic correlation of major pathologies of of shoulder instability 2008 Dec; 9(4): 187-193.

2. Gusmer PB, Potter HG, Schatz JA, Wickiewicz TL, Altchek DW, O'Brien SJ, Warren RFRadiology.Labral injuries: accuracy of detection with unenhanced MR imaging of the shoulder.1996 Aug;200(2):519-24

3. MA Zacchilli et al. Epidemiology of Shoulder Dislocations Presenting to 
Emergency Departments in the United States. J Bone Joint Surg Am : 2010; 92 (3), 542-549.

4. Bashir J, Sherman A, Lee H, Kaplan L, Hare JM. Mesenchymal stem cell therapies in the treatment of musculoskeletal diseases. PM R. 2014;6(1):61-9.

5. Lenza $\quad \mathrm{M}^{1}$, Buchbinder $\mathrm{R}$, Takwoingi Y, Johnston RV, Hanchard NC, Faloppa F 2013 MR Arthrography of the Labral Capsular Ligamentous Complex in the Shoulder: Imaging Variations and Pitfalls 2000.

6. de Jesus JO, Parker L, Frangos AJ, et al:Accuracy of MRI, MR arthrography, and ultrasound in the diagnosis of rotator cuff tears: a meta-analysis. AJR Am J Roentgenol, 2009;192:1701-1707.

7. SJ Kim, SH Kim, SK Lee, JW Seo, YM Chun: Arthroscopic repair of massive contracted rotator cuff tears: aggressive release with anterior and posterior interval slides do not improve cuff healing and integrity: J Bone Joint Surg Am, 2013, Aug 21; 95 (16): 1482 -1488.

8. Cluett J., Hill-Sachs Injury: Damage to the shoulder joint as result of dislocation, Orthopedics, 2013.

9. Waldt S, Burkart A, Imhoff AB, Bruegel M, Rummeny EJ, Woertler K. Anterior shoulder instability: Accuracy of MR arthrography in the classification of anterior labroligamentous injuries. Radiol. 2005;237:578-83

10. Palmer H, Jhankaria B, Maheshwari M, Singrakhia M, Shanbag S, Chawla A, Deshpande SJ Postgrad Med.Magnetic resonance arthrography in recurrent anterior shoulder instability as compared to arthroscopy: a prospective comparative study. 2002 Oct-Dec;48(4):270-3.

11. Bents RT, Skeete KD J Shoulder Elbow Surg. The correlation of the Buford complex and SLAP lesions 2005 Nov-Dec; 14(6):565-9. 